\title{
Comparación de dos técnicas en el manejo quirúrgico de la recesión radicular utilizando matriz dérmica acelular. Reporte de un caso
}

\section{Comparison of two surgical handling techniques for gingival recession using an acellular dermal matrix. A case report}

\author{
Juárez Membreño IA*
}

\section{RESUMEN}

Se han descrito, en cirugía plástica periodontal, diferentes procedimientos y materiales quirúrgicos con el propósito del tratamiento de recesión gingival $(R G)$, el incremento del espesor y ancho de encía adherida. Uno de los materiales empleados es la matriz dérmica acelular (MDA), aloinjerto de base biológica que de forma similar al tejido conectivo se incorpora a los tejidos periodontales, manteniendo la integridad estructural y la revascularización. Las técnicas para MDA puede ser un colgajo desplazado coronal (CDC) con incisiones verticales o la técnica quirúrgica en sobre o túnel modificado mediante el levantamiento desde el interior de las papilas permitiendo mayor movilización coronal del tejido y un desplazamiento coronal del colgajo. El objetivo del reporte de caso fue comparar las dos técnicas anteriores con un seguimiento de seis meses.

El caso clínico demostró concordancia con la evidencia de los estudios científicos que afirma éxito para tratar cobertura de recesiones con ganancia de tejido queratinizado, aumento de espesor de encía adherida, mejorías clínicas y resultados estéticos independiente de la técnica quirúrgica empleada en el uso de MDA.

Palabras clave: Matriz dérmica acelular (MDA), recesión gingival (RG), colgajo desplazado coronal (CDC), cobertura radicular (CR).

\section{SUMMARY}

Different surgical procedures and materials have been described with the purpose of carrying out gingival recession treatments (GR), the increase of thickness and width of adhered gum. One of the materials used is the acellular dermal matrix (ADM) biological base allograft which, like a connective tissue, is added to the periodontal tissues, keeping the structural integrity and re vascularization. The ADM techniques can be either a coronal displaced flap (CDF) with vertical incisions or a surgical technique in a kind of pocket or tunnel modified by means of a lifting from inside the papillae allowing a higher tissue coronal movement and a flap coronal displacement. The case report objective was to compare both techniques with a post six-month follow up. The clinical case showed concordance with the evidence of scientific studies which confirms the success for treating recession cover with a gain of keratinized tissue, increase of added gum thickness, better clinical improvements and aesthetic results regardless the surgery technique used when using ADM.

Key words: Acellular dermal matrix (ADM), gingival recession (RG), coronal displaced flap (CDC), root cover (RC).

Fecha de recepción: 20 de noviembre de 2013.

Aceptado para publicación: 27 de enero de 2014.

* Especialista en Periodoncia. Profesor Adjunto, Cátedra de Periodoncia. Instituto de Odontoestomatología. Escuela de Odontología. Facultad de Medicina. Universidad Austral de Chile.

Juárez Membreño I. Comparación de dos técnicas en el manejo quirúrgico de la recesión radicular utilizando matriz dérmica acelular. Reporte de un caso. Av. Odontoestomatol 2014; 30 (4): 187-194. 


\section{INTRODUCCIÓN}

La cirugía plástica periodontal se define como los procedimientos quirúrgicos que tienen el objetivo de prevenir, corregir o eliminar las deformidades de la mucosa alveolar entre las cuales se encuentra la morfología, posición, dimensión de la encía y tejido óseo que rodean al diente o al implante. Una de las indicaciones más comunes es el tratamiento de la recesión gingival (RG) (1-3), que es la exposición radicular debido a la migración de los tejidos periodontales hacia apical de la unión cemento esmalte (4-6), puede ser localizada o generalizada con una o más superficies comprometidas y su etiología está asociada a factores anatómicos, fisiológicos y patológicos (7).

En periodoncia se puede elegir entre diferentes técnicas quirúrgicas para lograr resultados óptimos y predecibles en el aumento de encía adherida (1, 2, 4, 7, 8), siendo objeto de innumerables esfuerzos y proyectos de investigación guiados al establecimiento y selección de técnicas quirúrgicas y materiales que permitan lograr de una manera predecible y no invasiva, el restablecimiento de tejidos periodontales compatibles con salud, función y estética periodontal (9).

Muchas modalidades quirúrgicas han sido utilizadas para alcanzar la cobertura radicular (CR) $(1,3)$. En la década de los años setenta, las técnicas más aceptadas fueron los colgajos desplazados coronalmente (CDC), lateralmente e injertos gingivales libres. Durante los años ochenta se introdujo el injerto de tejido conectivo subepitelial, aumentando la predictibilidad de cobertura para áreas mayores de exposición radicular (3), pero con las desventajas en el malestar postoperatorio y en la obtención de un sitio quirúrgico adicional (10).

En décadas recientes, se han descrito diferentes procedimientos quirúrgicos con el propósito del tratamiento de RG y el incremento del espesor y ancho de encía adherida (10). En los años noventa fue introducido el aloinjerto de matriz dérmica acelular (MDA) para ser utilizado en reemplazo del autoinjerto de tejido conectivo $(3,4,11)$.

La MDA es un aloinjerto de base biológica con indicaciones terapéuticas en cobertura de RG y aumento de encía adherida $(3,4,9,12-14)$, se procesa a partir de la piel obtenida de los donantes de tejidos humanos almacenados en bancos certificados. Los tejidos del donante se preparan mediante la eliminación de la epidermis y los componentes celulares de la piel reduciendo así la posibilidad de rechazo sobre el injerto. La MDA es entonces crioprotegida y rápidamente liofilizada, esto para preservar su integridad estructural y bioquímica (13-15), finalmente aumenta el espesor del tejido y a partir de un punto de vista histológico se adhiere a los tejidos periodontales de forma similar al tejido conectivo y sus cualidades no inmunogénicas hacen que sea viable el trasplante dermal, manteniendo la integridad estructural y la revascularización $(3,13)$.

Las técnicas para MDA puede ser un CDC que aumenta la probabilidad de obtener $\mathrm{CR}$ en recesiones clase I y II de Miller (16). Otra técnica que ha sido utilizada es el colgajo tipo sobre o túnel, descrita por Allen en $1994(17,18)$, con la característica inicial que la papila se deja intacta, además la ausencia de incisiones verticales tiene una tendencia a mejor estética y probablemente la principal ventaja de la técnica en sobre es que puede producir menor molestia en la zona receptora y utilizando MDA permite que sea un procedimiento quirúrgico mínimamente invasivo sin necesidad de obtener tejido palatino $(3,13)$. En años recientes se reportó la modificación de la técnica en sobre donde a través de una disección más profunda se libera el tejido gingival vestibular y mediante el levantamiento desde el interior de las papilas permite movilización coronal del tejido logrando mayor libertad en el desplazamiento coronal del colgajo (13).

Los reportes clínicos demuestran que la MDA es una alternativa para incrementar el espesor de encía queratinizada (19) y bajo un colgajo coronal ha demostrado éxito y posibilidad de obtener CR en un $100 \%$ de la recesión (13). El propósito de este reporte clínico es comparar dos técnicas quirúrgicas: el colgajo con desplazamiento coronal e incisiones verticales y la técnica de túnel o sobre modificada en el uso de la MDA.

\section{PRESENTACIÓN DEL CASO}

Paciente sexo femenino, 22 años de edad, chilena, sin enfermedades sistémicas, no fumadora. Acude 
a la Especialidad de Periodoncia ya que desea efectuar tratamiento de CR. Al examen clínico la paciente presenta PSR código global cero, ausencia de signos y síntomas de inflamación, adecuado control de higiene, periodonto fino y recesiones clase I de Miller en dientes 1.5, 1.4, 1.3 y 4.4 que se extiende 4 milímetros de la unión cemento esmalte (Fig. 1).

\section{Procedimiento quirúrgico}

Previo consentimiento informado, se toma la decisión de efectuar CR utilizando matriz dérmica acelular Alloderm y realizar en los dientes 1.5, 1.4, 1.3 la técnica de sobre modificado y en diente 4.4 la técnica de CDC con incisiones verticales. Se emplea anestesia local (lidocaína 2\%, epinefrina 1:100,000).

Para el diseño de la incisión quirúrgica en la técnica de sobre modificado, se utiliza mango universal en 360 grados con hoja de bisturí descartable mesial de 1.3 conservando la integridad de la papila interdentaria (Fig. 2), el bisturí de orban 1/2, para la incisión interna en el tejido interdentario y el periostótomo para lograr la modificación del sobre (Figs. 3 a 5). La incisión en diente 4.4 para el CDC con incisiones verticales se realiza con bisturí convencional, el bisturí de orban $1 / 2$ y el periostótomo para desplazamiento del colgajo (Figs. 6 a 8 ), luego se hace una

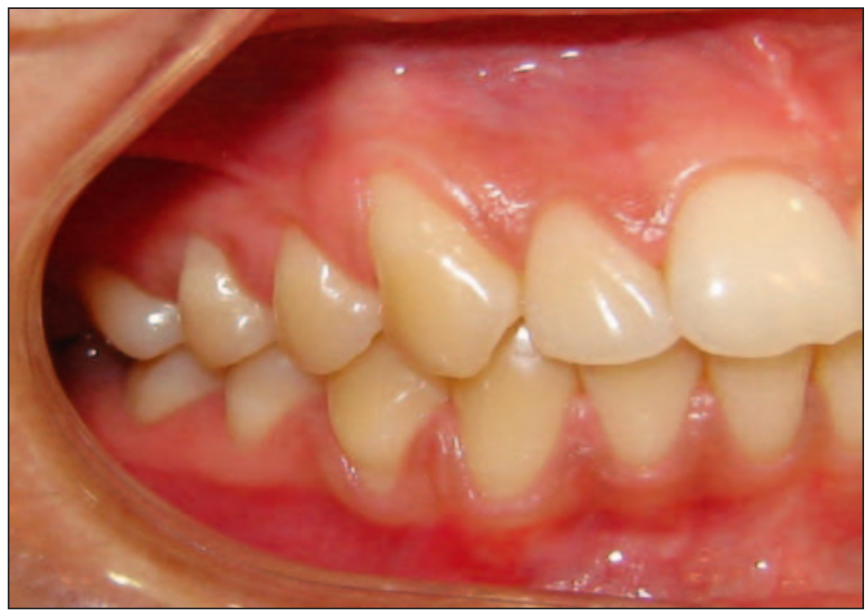

Fig. 1. Evaluación inicial. Presencia de recesiones gingivales clase I según Miller en 1.5, 1.4, 1.3 y 4.4 .

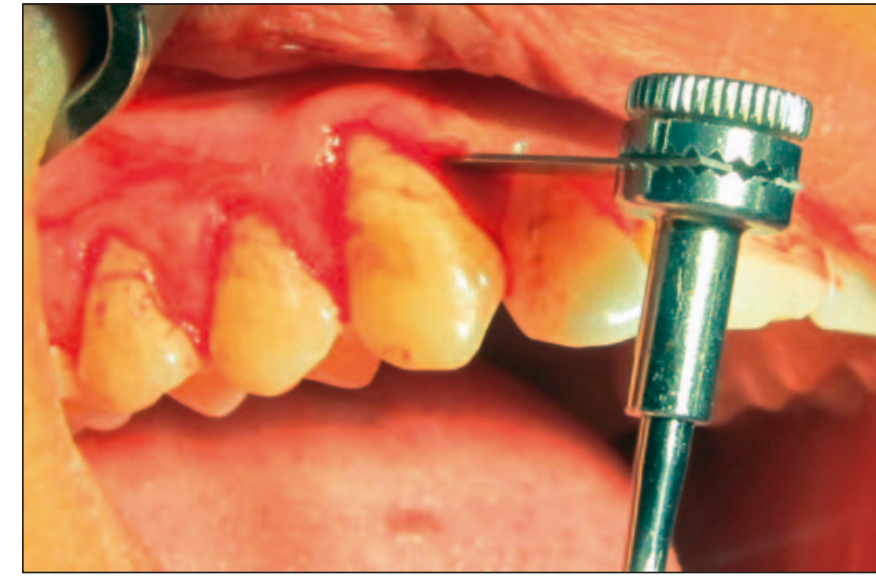

Fig. 2. Incisión con mango universal en 360 grados con el objetivo de lograr la introducción de la MDA desde mesial de 1.3 y además un mejor desplazamiento hacia coronal en la técnica de sobre o túnel.

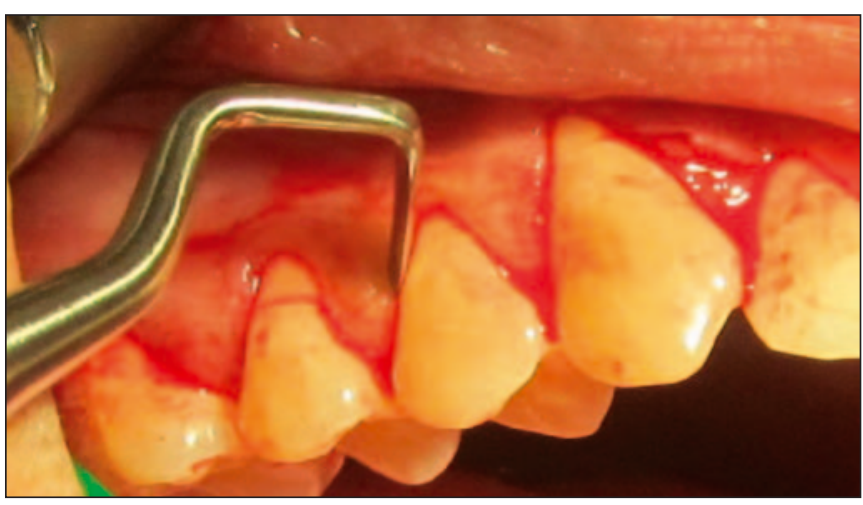

Fig. 3. Utilización del bisturí Orban en el área interproximal logrando un desplazamiento mayor del tejido gingival vestibular durante la técnica de sobre o túnel modificada.

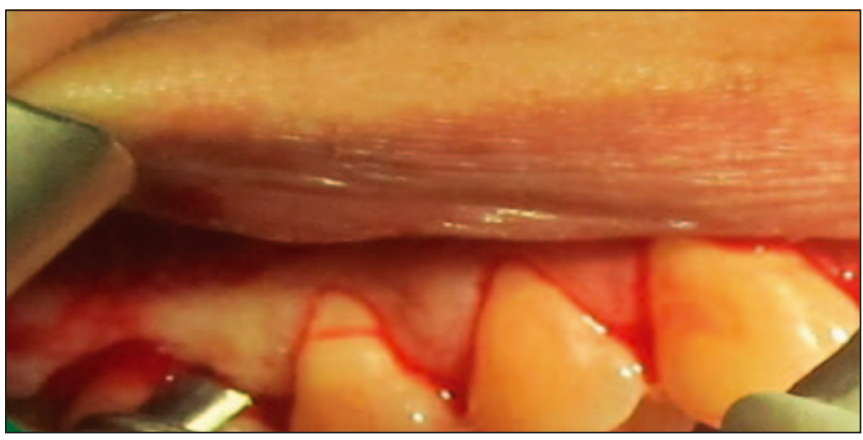

Fig. 4. Utilización del bisturí Orban en superficie distal de diente 1.5 facilitando el desplazamiento hacia coronal durante la elaboración de técnica de sobre o túnel modificada. 


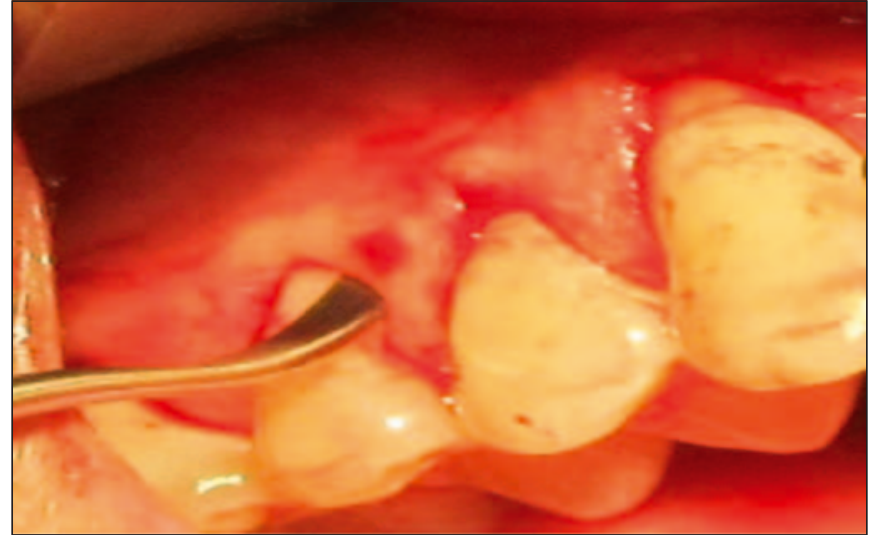

Fig. 5. Uso del periostótomo para lograr la modificación de la técnica en sobre.

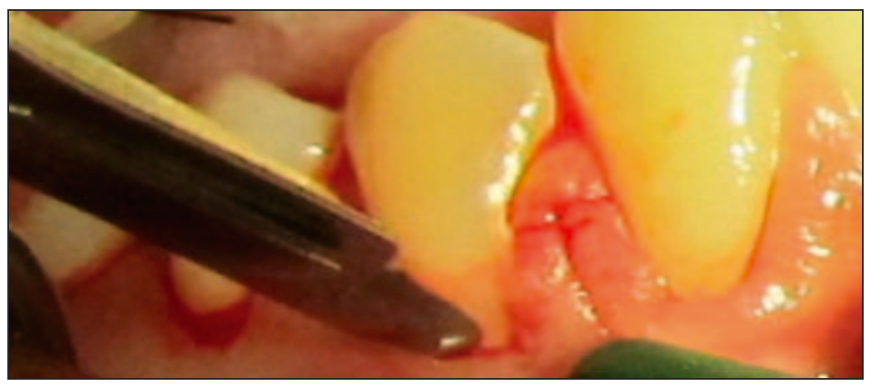

Fig. 6. Incisión en diente 4.4 a través del CDC con incisiones verticales, conservando la integridad de las papilas interdentarias.

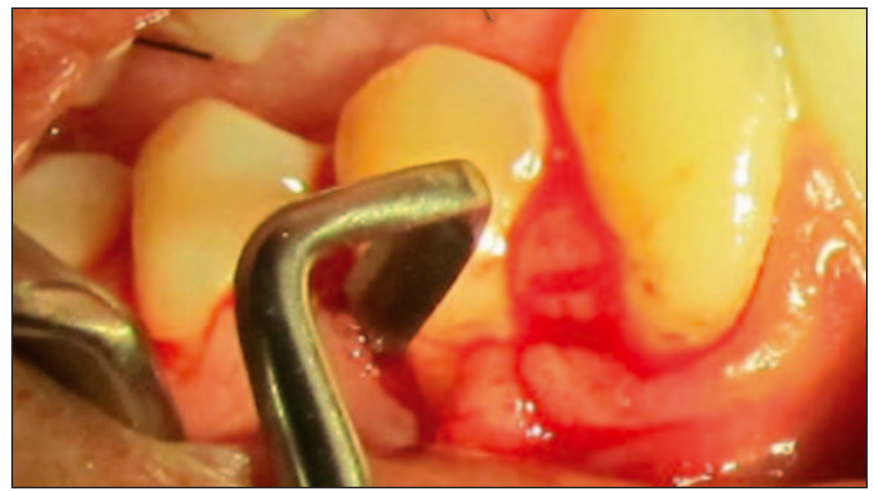

Fig. 7. Utilización de bisturí de orban en 4.4, facilitando el diseño del CDC con incisiones verticales. Se aprecia la integridad de las papilas durante la incisión.

desepitelización papilar (Figs. 9 y 10), se procede a la toma y colocación del injerto (Figs. 11 y 12), la sutura de la MDA empleando material sintético reab-

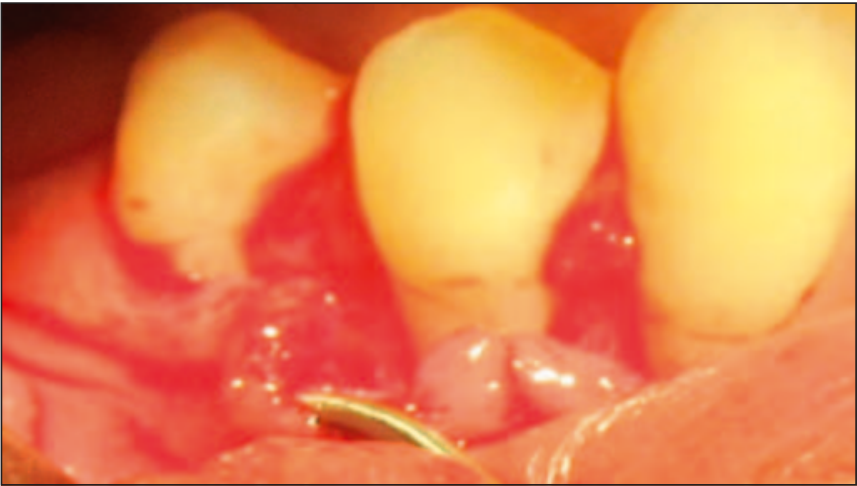

Fig. 8. Desplazamiento total del colgajo a través del periostótomo en la recesión de diente 4.4.

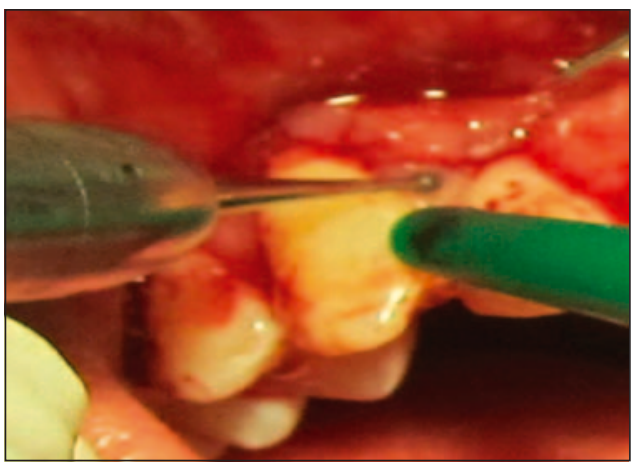

Fig. 9

Desepitelización de papilas superiores.
Fig. 10. Desepitelización de papilas inferiores.

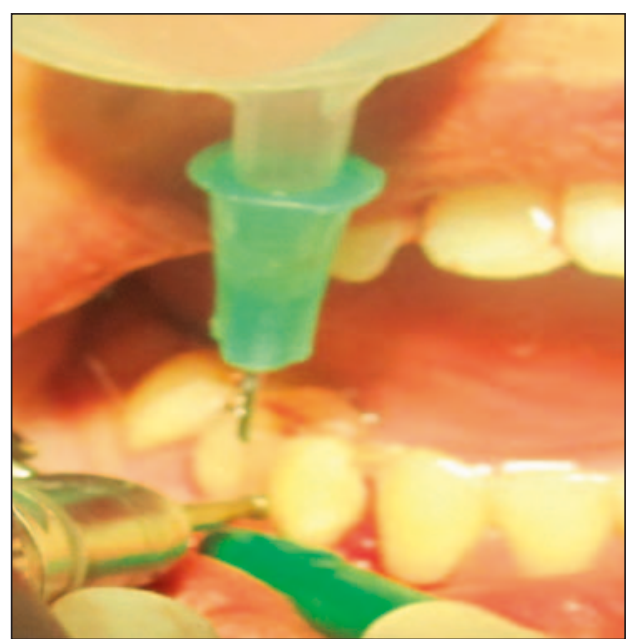

sorbible polysorb 6-0* lactomer 9-1, (0.7 metric) 45 cm SL-5688 (Fig. 13) y finalmente la sutura del colgajo en sobre modificado superior y el CDC con in- 


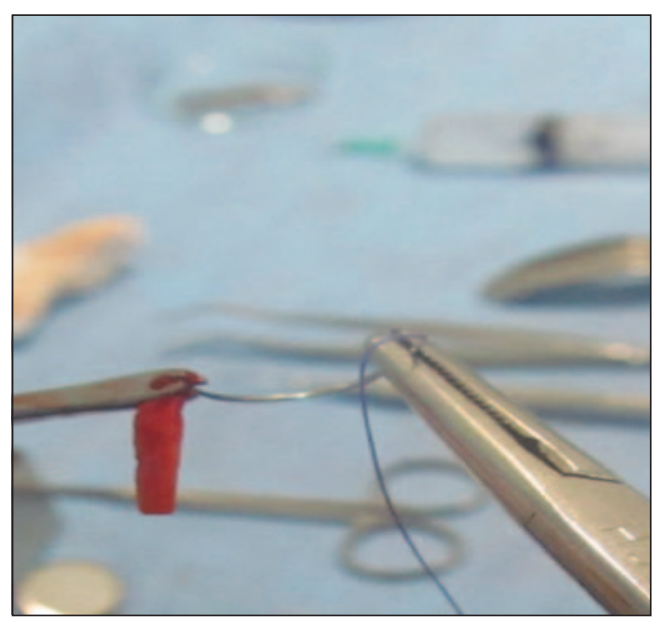

Fig. 11. Toma de la matriz dérmica acelular.

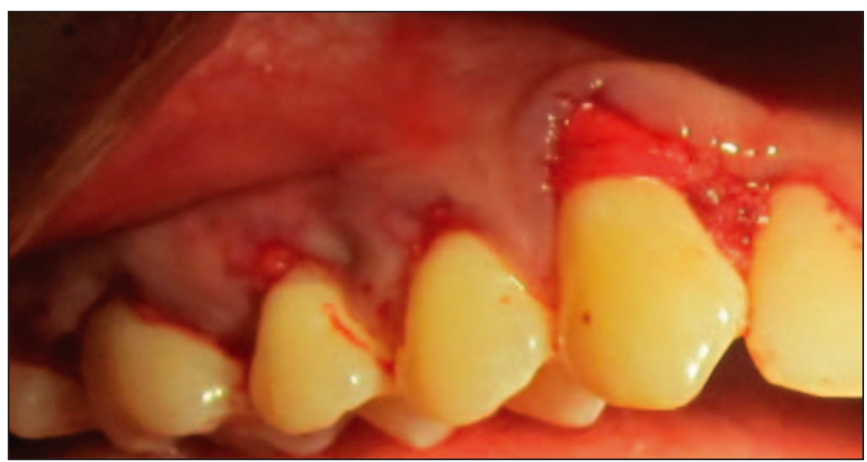

Fig. 12. Colocación de la matriz dérmica acelular.

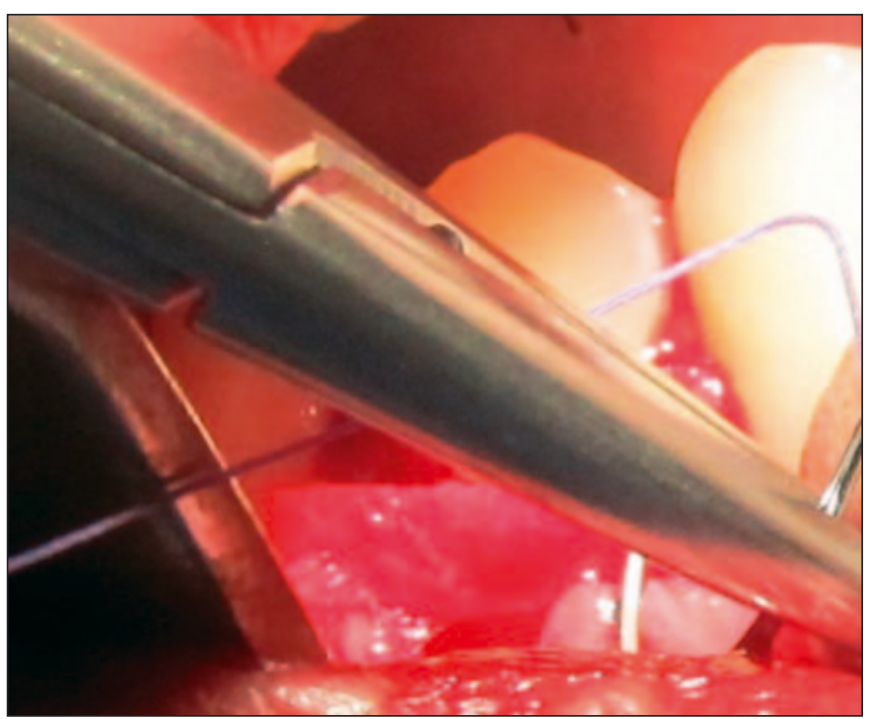

Fig. 13. Sutura de la matriz dérmica acelular a través de sutura reabsorbible. cisiones verticales inferior con material no reabsorbible Mononylon Ethilon 3-0 (2 metric) $75 \mathrm{~cm}$ triangular SC-24 (Figs. 14 y 15).

\section{Observaciones clínicas}

La curación de los tejidos fue completa. Durante la evaluación clínica no existió exposición de la MDA. A las tres semanas la encía del sitio quirúrgico donde se empleó la técnica de sobre modificado se encontraba un poco edematosa pero se observó cobertura total de las recesiones y aumento en el espesor de tejido queratinizado al emplear ambas técnicas quirúrgicas (Fig. 16). Posteriormente a los seis meses se logró apariencia normal de los tejidos periodontales, ausencia de inflamación, mantenimiento de la cobertura total de recesiones y aumento del espesor de tejido queratinizado empleando ambas técnicas (Fig. 17), además profundidad de sondaje de un

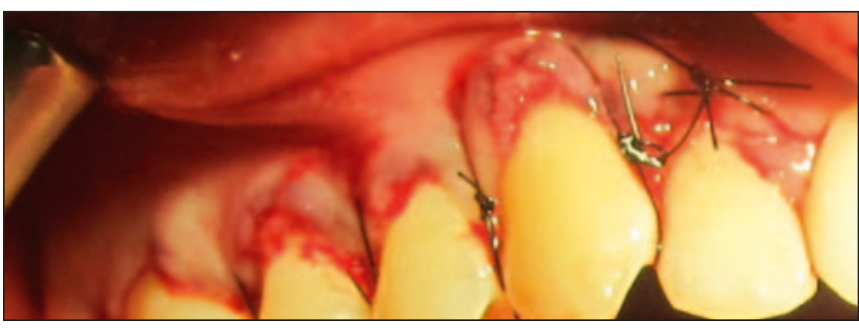

Fig. 14. Sutura final arco dental superior, logrando la técnica de sobre modificada.

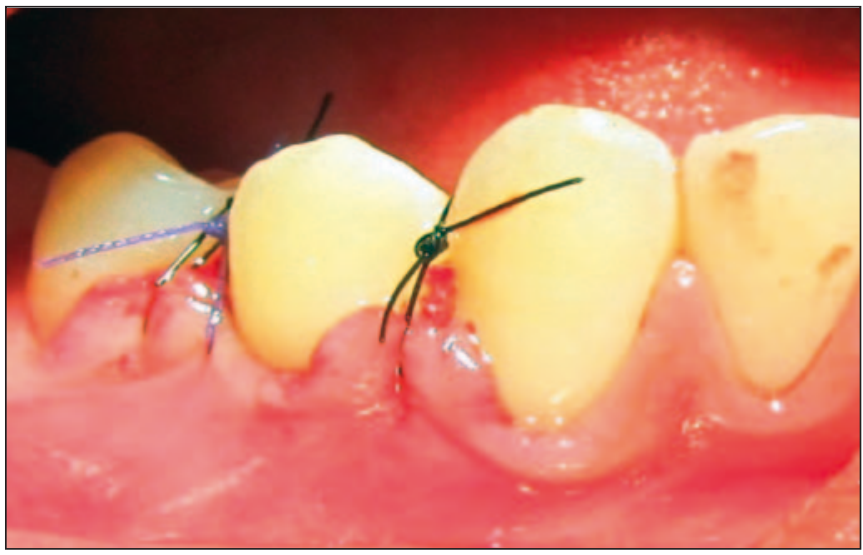

Fig. 15. Sutura diente 4.4 logrando la cobertura de la matriz dérmica acelular y el CDC. 


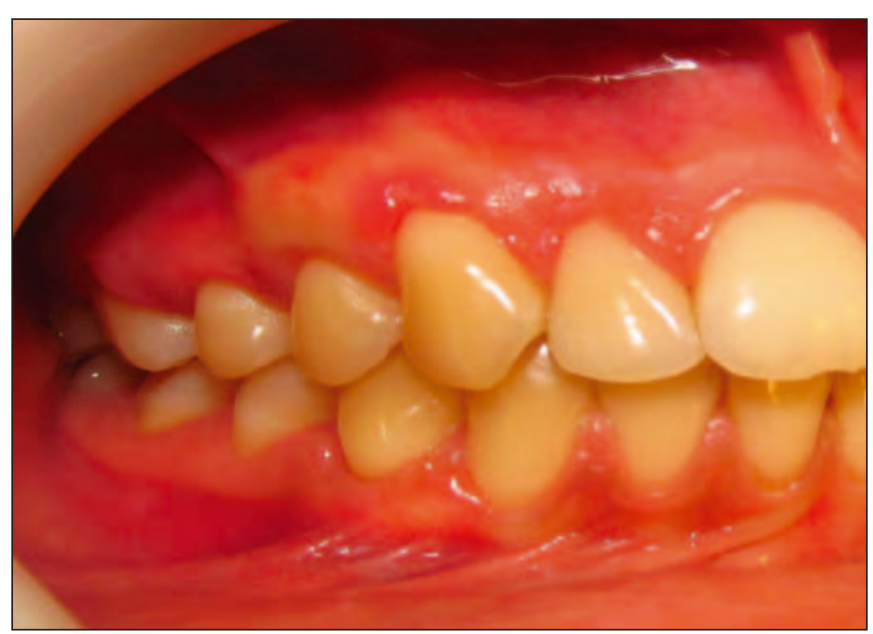

Fig. 16. Control a las 3 semanas después de la cirugía periodontal. Se observa cobertura total de recesiones y aumento de tejido queratinizado. Leve edema en diente 1.3.

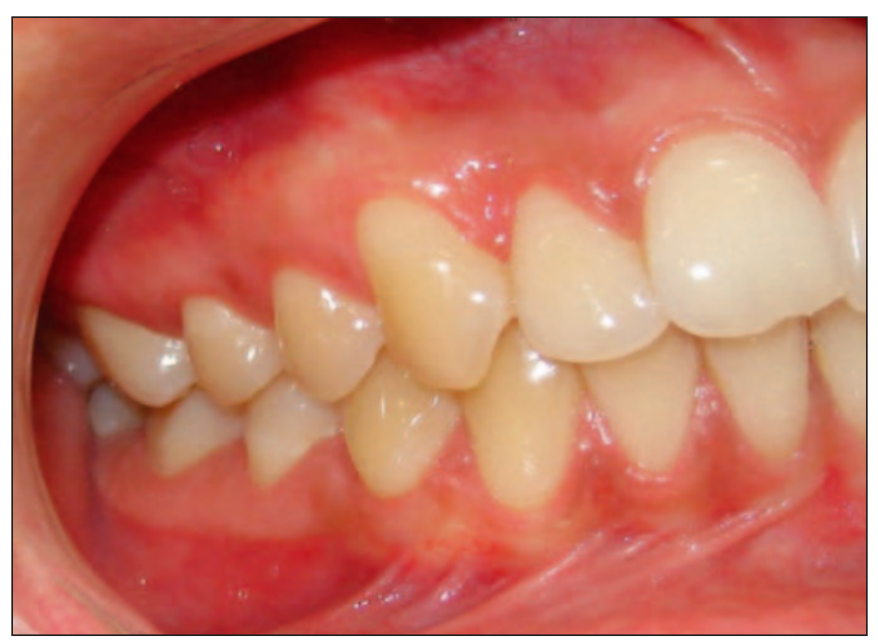

Fig. 17. Control a los 6 meses después de la cirugía periodontal, se logra cobertura total de recesiones y aumento de espesor de tejido queratinizado tanto en la técnica de sobre modificada superior y el colgajo desplazado coronal inferior con incisiones verticales.

milímetro compatible con surco gingival y salud periodontal.

\section{DISCUSIÓN}

Durante la comparación de las dos técnicas quirúrgicas empleando MDA se confirmó que es un aloinjerto adecuado, coincidiendo con publicaciones que lo recomiendan para el aumento de espesor de tejido queratinizado $(3,4)$, mejorías clínicas y resultados estéticos $(3,4,8,9,11,14,20,21)$.

Con respecto a MDA y las diferentes técnicas quirúrgicas ha sido reportado que el uso combinado de la técnica tipo sobre modificado y la MDA para la ganancia de tejido queratinizado y CR es una excelente opción clínica (3), disminuye complicaciones y molestias en el periodo postoperatorio (13) y logra recubrimiento radicular total (9), similar al caso clínico presentado donde se obtuvo éxito clínico en los dientes 1.5, 1.4 y 1.3. Por lo tanto el uso de la MDA empleando la técnica de sobre en recesiones múltiples puede ser una alternativa viable a la técnica tradicional con injerto conectivo especialmente y se debe considerar en pacientes con limitaciones de tejido conectivo (9). De igual forma el uso de MDA reduce la $R G$ y logra un mayor espesor gingival con la técnica de $\operatorname{CDC}(22,23)$, con resultados mejor a corto plazo (14) en recesiones de Miller clases I y II en dientes individuales a los 6 meses (9).

En el caso clínico que se presentó, se concuerda con el planteamiento anterior donde se demostró durante el seguimiento que existió éxito en $\mathrm{CR}$, ganancia de tejido queratinizado y aumento de espesor de tejido gingival utilizando ambas técnicas.

En lo concerniente al logro de resultados estéticos, se ha descrito que usando MDA se obtiene armonía con los dientes vecinos y un incremento en encía queratinizada (24), resultado que también concuerda con el caso clínico presentado. La MDA es un procedimiento predecible en cirugía plástica periodontal (25) y se puede emplear tanto la técnica de sobre modificado como CDC.

\section{CONCLUSIÓN}

Independiente de la técnica a utilizar, la MDA puede ser empleada con éxito en CR y ganancia de tejido queratinizado. Además, en concordancia con la evidencia de los estudios científicos, se afirma que la MDA proporciona éxito para tratar cobertura de recesiones con ganancia de tejido queratinizado, mejorías clínicas, resultados estéticos evitando la necesidad de obtener injerto conectivo del paladar; por lo 
tanto, se sugiere la realización de estudios clínicos aleatorios sobre los procedimientos en el uso de MDA.

\section{BIBLIOGRAFÍA}

1. Ardila C. Hipersensibilidad dentinal: Una revisión de su etiología, patogénesis y tratamiento. Av Odontoestomatol 2009;25:137-46.

2. Mahajan A, Dixit J, Verma U. A Patient-Centered Clinical Evaluation of Acellular Dermal Matrix Graft in the Treatment of Gingival Recession Defects. J Periodontol 2007;78:2348-55.

3. Rivera R, Castillo R, Málaga L. Tratamiento de Recesiones Gingivales Múltiples con Matriz Dérmica Acelular (Alloderm ${ }^{\circledR}$ ): Resultados a Corto Plazo en Zona Estética. Rev Clin Periodoncia Implantol Rehábil Oral 2009;2:182-86.

4. Shin S, Cueva M, Kerns D. et al. A Comparative Study of Root Coverage Using Acellular Dermal Matrix With and Without Enamel Matrix Derivative. J Periodontol 2007;78:411-21.

5. Barker T, Cueva M, Rivera-Hidalgo F. et al. A Comparative Study of Root Coverage Using Two Different Acellular Dermal Matrix Products. J Periodontol 2010;81:1596-3.

6. Kuru B, Yýldýrým S. Treatment of Localized Gingival Recessions Using Gingival Unit Grafts: A Randomized Controlled Clinical Trial. J Periodontol 2013;84:41-50.

7. Soto M, Weber B, Fuentes R, Olate S. Manejo quirúrgico de la recesión radicular con injerto de tejido conectivo. Reporte de un caso. Int $\mathrm{J}$ Odontostomat 2009;3:103-8.

8. Wei P, Laurell L, Geivelis M, Lingen M, Maddalozzo D. Acellular Dermal Matrix Allografts to Achieve Increased Attached Gingiva. Part 1. A Clinical Study. J Periodontol 2000;71:1297-5.

9. Durán J, Alarcón C, Velásquez D. Aplicación de biomateriales de base biológica, moléculas bio- activas e ingeniería de tejidos en cirugía plástica periodontal Una revisión. Rev Clin Periodoncia Implantol Rehabil Oral 2012;5:142-9.

10. Gapski R, Allen C, Wang H. Acellular Dermal Matrix for Mucogingival Surgery: A Meta-Analysis. J Periodontol 2005;76:1814-22.

11. Tal H, Moses O, Zohar R, Meir H, Nemcovsky C. Root Coverage of Advanced Gingival Recession: A Comparative Study Between Acellular Dermal Matrix Allograft and Subepithelial Connective Tissue Grafts. J Periodontol 2002;73:1405-11.

12. Cummings L, Kaldahl W, Allen E. Histologic Evaluation of Autogenous Connective Tissue and Acellular Dermal Matrix Grafts in Humans. J Periodontol 2005;76:178-86.

13. Papageorgakopoulos G, Greenwell H, Hill M, Vidal R, Scheetz J. Root Coverage Using Acellular Dermal Matrix and Comparing a Coronally Positioned Tunnel to a Coronally Positioned Flap Approach. J Periodontol 2008;79:1022-30.

14. Cortellini P, Pini Prato G. Coronally advanced flap and combination therapy for root coverage. Clinical strategies based on scientific evidence and clinical experience. Periodontol 2000 2012;59:158-84.

15. Maia L, Novaes Jr. A, Souza S, Grisi M, Taba Jr M. et al. In Vitro Evaluation of Acellular Dermal Matrix as a Three-Dimensional Scaffold for Gingival Fibroblasts Seeding. J Periodontol 2011; 82:293-1.

16. Cairo F, Pagliaro U, Nieri M. Treatment of gingival recession with coronally advanced flap procedures: a systematic review. J Clin Periodontol 2008;35 (Supl 8):136-62.

17. Allen A. Use of the supraperiosteal envelope in soft tissue grafting for root coverage. Rationale and technique. Int J Periodontics Restorative Dent 1994; 14:216-27.

18. Allen A. Use of the supraperiosteal envelope in soft tissue grafting for root coverage Clinical 
results. Int J Periodontics Restorative Dent 1994; 14:302-15.

19. Buduneli E, Ílgenli T, Buduneli N, Ôzdemir F. Acellular dermal matrix allograft used to gain attached gingiva in a case of epidermolysis bullosa. J Clin Periodontol 2003;30:1011-5.

20. Palmer R, Cortellini P. Periodontal tissue engineering and regeneration: Consensus Report of the Sixth European Workshop on Periodontology. J Clin Periodontol 2008;35 (Supl 8):83-6.

21. Calefi P, Sorgini D, Borie E, Toniollo M, Barros L. et al. Tratamiento periodontal quirúrgico en rehabilitación oral estética: reporte de caso clínico. Int J Odontostomat 2012;6:363-8.

22. Andrade P, Felipe M, Novaes A, Souza S, Taba M, Palioto D, Grisi M. Comparison between two surgical techniques for root coverage with an acellular dermal matrix graft. J Clin Periodontol 2008;35:263-9.
23. De Queiroz A, Sallum A, Casati M, Nociti F, Sallum E. A two-year prospective study of coronally positioned flap with or without acellular dermal matrix graft. J Clin Periodontol 2006;33:683-9.

24. Tal H. Subgingival Acellular Dermal Matrix Allograft for the Treatment of Gingival Recession: A Case Report. J Periodontol 1999;70:1118-24.

25. Henderson R, Greenwell H, Drisko C, Regenniter F, Lamb J. et al. Predictable Multiple Site Root Coverage Using an Acellular Dermal Matrix Allograft. J Periodontol 2001;72:571-82.

\section{CORRESPONDENCIA}

Israel Antonio Juárez Membreño Profesor Adjunto, Cátedra de Periodoncia. Instituto de Odontoestomatología. Escuela de Odontología Facultad de Medicina. Universidad Austral de Chile Rudloff 1640. Valdivia. Chile Email: juarezuach@gmail.com 